\title{
Article \\ Effectiveness of Video-Assisted Thoracoscopic Surgery with Bullectomy and Partial Pleurectomy in the Treatment of Primary Spontaneous Pneumothorax-A Retrospective Long-Term Single-Center Analysis
}

\author{
Stephen Fung ${ }^{1}\left(\mathbb{D}\right.$, , Hany Ashmawy ${ }^{1}\left(\mathbb{D}\right.$, Sami-Alexander Safi ${ }^{1} \mathbb{D}$, Matthias Schauer ${ }^{2}$, Andreas Krieg ${ }^{1}{ }^{(D)}$, \\ Anja Schauer ${ }^{1}$, Marius Kivilis ${ }^{1}$, Farid Ziayee ${ }^{3} \mathbb{D}$, Alexander Rehders ${ }^{1}$, Levent Dizdar ${ }^{1,+}$ \\ and Wolfram-Trudo Knoefel ${ }^{1, *,+}$
}

check for updates

Citation: Fung, S.; Ashmawy, H.; Safi, S.-A.; Schauer, M.; Krieg, A.; Schauer,

A.; Kivilis, M.; Ziayee, F.; Rehders, A.;

Dizdar, L.; et al. Effectiveness of

Video-Assisted Thoracoscopic

Surgery with Bullectomy and Partial

Pleurectomy in the Treatment of

Primary Spontaneous

Pneumothorax-A Retrospective

Long-Term Single-Center Analysis.

Healthcare 2022, 10, 410. https://

doi.org/10.3390/healthcare10030410

Academic Editor: Wilhelm Mistiaen

Received: 13 January 2022

Accepted: 19 February 2022

Published: 22 February 2022

Publisher's Note: MDPI stays neutral with regard to jurisdictional claims in published maps and institutional affiliations.

Copyright: (C) 2022 by the authors. Licensee MDPI, Basel, Switzerland. This article is an open access article distributed under the terms and conditions of the Creative Commons Attribution (CC BY) license (https:// creativecommons.org/licenses/by/ $4.0 /)$.
1 Department of Surgery, University Hospital Duesseldorf and Heinrich-Heine-University Duesseldorf, 40225 Duesseldorf, Germany; stephen.fung@med.uni-duesseldorf.de (S.F.);

hany.ashmawy@med.uni-duesseldorf.de (H.A.); sami-alexander.safi@med.uni-duesseldorf.de (S.-A.S.); andreas.krieg@med.uni-duesseldorf.de (A.K.); anjamaria.schauer@med.uni-duesseldorf.de (A.S.); marius.kivilis@med.uni-duesseldorf.de (M.K.); rehders@med.uni-duesseldorf.de (A.R.); levent.dizdar@med.uni-duesseldorf.de (L.D.)

2 Department of General and Thoracic Surgery, Augusta Hospital Duesseldorf, 40472 Duesseldorf, Germany; schauer@rocketmail.com

3 Department of Radiology, University Hospital Duesseldorf and Heinrich-Heine-University Duesseldorf, 40225 Duesseldorf, Germany; farid.ziayee@med.uni-duesseldorf.de

* Correspondence: knoefel@hhu.de; Tel.: +49-211-81-17350; Fax: +49-211-81-17359

+ These authors contributed equally to this work.

\begin{abstract}
Background: Video-assisted thoracoscopic surgery (VATS) with bullectomy and partial pleurectomy (VBPP) is an increasingly used and well-established surgical treatment for primary spontaneous pneumothorax (PSP). However, reports on its effectiveness and long-term outcomes are limited. The aim of this study was to assess and compare long-term recurrence rates following VBPP and chest tube (CT) treatment and to identify potential risk factors for disease recurrence in patients with PSP. Methods: A total of 116 patients treated either by VBPP or CT were included in this study. Long-term recurrence rates and associations between clinical parameters and recurrence of pneumothorax were analyzed. Results: Sixty-two patients (53.4\%) underwent VBPP, whereas $54(46.6 \%)$ patients underwent CT treatment only. During a median follow-up period of 76.5 months, VBPP patients experienced a significantly lower recurrence rate compared to CT patients (6/62 vs. 35/54; $p<0.0001)$. CT treatment (VBPP vs. CT; $p<0.001$ ) and a large initial pneumothorax size (Collins $<4$ vs. Collins $\geq 4 ; p=0.018$ ) were independent risk factors for pneumothorax recurrence. Conclusion: VBPP is an effective and safe surgical treatment for PSP. Therefore, patients with a large pneumothorax size might benefit from VBPP, as they are at high risk for disease recurrence.
\end{abstract}

Keywords: VATS bullectomy; partial pleurectomy; chest tube; recurrence; PSP

\section{Introduction}

According to the German S3 guidelines, primary spontaneous pneumothorax (PSP) describes the presence of air without preceding trauma or underlying pulmonary disease within the pleural space of patients under 45 years of age [1]. The incidence of PSP has been reported with approximately 1-9.8 and 7-24 cases per 100,000 individuals per year in females and males, respectively [2,3]. In most cases, PSP results from the rupture of subpleural blebs and bullae, predominantly in young, thin males [4,5]. Despite unknown etiology of PSP, associated risk factors for its occurrence and recurrence, such as male sex, tall stature, nicotine abuse, size of pneumothorax, and a family history of pneumothorax, have been reported [6-9]. 
According to the current guidelines, the initial treatment algorithm depending on the patient's clinical condition includes observation, oxygen supplementation, needle aspiration, and chest tube drainage $[1,10,11]$. Although PSP often resolves by observational conservative approach or by chest tube drainage [12,13], high rates of recurrence after these treatment modalities have been described [14-17]. For cases with ipsilateral recurrence of PSP and those with persistent air leak following chest tube (CT) treatment, the guidelines $[1,10,11]$ recommend video-assisted thoracoscopic surgery (VATS). In previous studies, VATS with bullectomy alone was demonstrated to have recurrence rates up to $20 \%[18,19]$. When combined with pleurectomy, the short- and medium-term recurrence rates were reported to reduce to 1-6\% [20-22]. For long-term results, only a few studies are described in the literature [23-25].

Therefore, the primary aim of this study was to evaluate the long-term recurrence rates following VBPP and to compare these results with those of patients successfully treated by CT only at our institution. Secondly, we analyzed underlying clinical features to determine potential risk factors for pneumothorax recurrence in our PSP cohort.

\section{Materials and Methods}

We retrospectively reviewed data of 120 patients with primary spontaneous pneumothorax (PSP) treated either by VATS bullectomy with partial pleurectomy (VBPP) or by chest tube (CT) only between January 2008 and December 2020 in our institution. Patient demographics, including age, sex, body mass index (BMI), smoking status, length of hospital stay (LOS), duration of surgery, time until recurrence, treatment modality, complications, and size of the pneumothorax, were retrieved from medical records. The size of the pneumothorax was assessed using the regression formula derived from the method of Collins [26]. According to the actual German S3 guidelines for management of spontaneous pneumothorax and post-interventional pneumothorax, a spontaneous pneumothorax (SP) is considered as large when the sum of the interpleural distances derived from Collins method is $\geq 4 \mathrm{~cm}$ [1]. Hence, in this study, we considered a spontaneous pneumothorax to be large at a size of $\geq 4 \mathrm{~cm}$.

At first presentation of spontaneous pneumothorax (SP), identified patients received chest tube (CT) treatment. Patients who were initially successfully treated with CT during our study period were classified in the CT group. Indication for surgery (VBPP) included persistent air leak for more than 5 days following CT treatment $(n=20)$, first ipsilateral recurrent pneumothorax (recurrence of pneumothorax on the previously treated side, $n=32$ ), synchronous bilateral spontaneous pneumothorax $(n=8)$, and spontaneous hemopneumothorax $(n=2)$. Of note, patients who received VBPP for recurrence either underwent initial CT treatment at our institution before our study period or had received CT treatment at another hospital and subsequently presented in our institution with recurrence. Prior to surgery, a computer tomography of the chest was performed to detect any bullous disease. A team of three specialized thoracic surgeons (WTK, AS, AR) made indication for surgery. Patients with incomplete follow-up data and patients who received other treatment modalities (e.g., thoracotomy, apical pleurectomy (pleurectomy of the apex of the pleural cavity only), observation, needle aspiration) were excluded from this study. The primary objectives of this study were to assess and compare long-term recurrence rates after treatment with VBPP and CT in our institution and to identify potential risk factors for pneumothorax recurrence. Recurrence was described as an ipsilateral pneumothorax detected on a chest radiograph at presentation in our emergency room after surgical treatment by VBPP or chest tube drainage. The local ethic committee of the Heinrich-Heine University Clinic of Duesseldorf approved this study (study no.: 2020-1271).

\subsection{Surgical Technique: VATS Bullectomy with Partial Pleurectomy (VBPP)}

Our specialized team of thoracic surgeons (A.S., A.R., and W.T.K.) performed all surgical procedures and postoperative patient follow-up. All the patients were treated under general anesthesia with a double-lumen tube intubation and single-lung ventilation. 
After lateral positioning of the patient, video-assisted thoracoscopic surgery (VATS) was performed in the conventional two- or three-port approach. Initial thoracoscopy was undertaken for thorough inspection of the visceral and parietal pleura. Bullectomy was carried out when blebs or bullae were identified by wedge resection using an endoscopic stapling device (Autosuture GIA Universal; COVIDIEN ${ }^{\mathrm{TM}}$, Mansfield, MA, USA). Partial pleurectomy was performed beginning from the apex of the pleural cavity. During this procedure, the parietal pleura was carefully separated from the endothoracic fascia while sparing the region of the subclavian artery and vein to avoid injury of these structures. Pleurectomy was performed up to the 7th or 8th intercostal space in a blunt manner. After cautious hemostasis of the endothoracic fascia using electrocautery to reduce the risk of hemothorax, one 24-Fr. chest tube was inserted and connected to a digital underwater seal system (Thopaz+, Medela AG, Baar, Switzerland) with a suction of $-20 \mathrm{~mm}$ Hg. During postoperative care, the chest tube drain was removed when no clinical signs of air leak and a drain output of less than $200 \mathrm{~mL}$ after $24 \mathrm{~h}$ were evident. After chest tube removal, a chest radiograph was taken to verify full expansion of the lung. All the patients (VBPP and CT treated patients) received our standard postoperative medication regime of analgesia (nonopioid, orally or intravenously). The patients received either metamizole-natrium $1000 \mathrm{mg}$, paracetamol $1000 \mathrm{mg}$, or ibuprofen $600 \mathrm{mg}$ four times per day. In cases of persistent pain using the standard pain medication regime, we applied piritramide (opioid) $7.5 \mathrm{mg}$ intravenously every $4-6 \mathrm{~h}$ on patient request.

\subsection{Outpatient Care and Follow-Up}

One week after discharge, the patients visited our outpatient clinic for postoperative control and follow-up. These visits continued at a 3-month interval for one year. A chest radiograph was taken at each visit. The patients were advised to visit our emergency room at any time they had symptoms related to recurrent pneumothorax, such as dyspnea, chest pain, or cough. Recurrent pneumothorax was identified clinically in each case with a chest radiograph. For patients who recurred after CT or VBPP treatment, VBPP or re-VATS was performed, respectively. For long-term follow-up, patients were contacted and assessed with a questionnaire.

\subsection{Statistical Analysis}

All data were analyzed with the SPSS 25.0 software program (Statistical Package for Social Sciences; SPSS Inc., Chicago, IL, USA). Patients' data were expressed as numbers, mean or median. Continuous variables were compared using a Mann-Whitney U test, and the chi-square test was implemented for categorical data. Recurrence-free survival (RFS) was defined as the period between initial treatment by surgery (VBPP) or chest tube (CT) and ipsilateral recurrent pneumothorax. Kaplan-Meier curves were generated and evaluated with the log-rank test (Mantel-Cox test), and hazard ratios (HRs) were estimated with $95 \%$ confidence intervals (CIs). For multivariate analysis, all variables were included in a logistic regression analysis. Statistical significance was considered at $p<0.05$.

\section{Results}

Between January 2008 and December 2020, 120 patients with primary spontaneous pneumothorax (PSP) were treated either by VATS-bullectomy with partial pleurectomy (VBPP) or by chest tube (CT) in our institution. Four patients were lost during followup and were excluded from this analysis. A total of 116 patients with a median age of 24 years (range 18-41) were included in this study. Sixty-two patients underwent VBPP, whereas 54 patients received CT treatment. The clinical characteristics of the patients are summarized in Table 1.

At presentation, the mean pneumothorax sizes estimated according to Collins method were $13.4 \mathrm{~cm}$ and $13.9 \mathrm{~cm}$ in the VBPP and CT groups, respectively, indicating a large pneumothorax size for both patient cohorts. Clinical variables, such as age, gender, BMI, and pneumothorax size and chest tube duration, were similar in both groups (Table 1). 
Three patients suffered a hemothorax in the VBPP group. Of these patients, two were successfully treated conservatively, while one patient underwent re-VATS. Likewise, 24 patients suffered a prolonged air leak following VBPP. In all the cases, the patients were successfully treated conservatively. Significant differences were found between the two groups in terms of patients' smoking behaviour and the length of hospital stay (LOS). The proportion of smokers was significantly higher and the LOS significantly longer in the VBPP group compared to the CT group. However, it must be considered that the LOS in the VBPP group was significantly prolonged by the preoperative period until surgery was performed (mean of 4.1 days).

Table 1. Clinical characteristics of the VBPP and CT group.

\begin{tabular}{|c|c|c|c|}
\hline Variables & $\begin{array}{c}\text { VBPP } \\
n=62(\%)\end{array}$ & $\begin{array}{c}\text { CT } \\
n=54(\%)\end{array}$ & $p$-Value \\
\hline \multicolumn{4}{|l|}{ Gender } \\
\hline Male & $48(77.4)$ & $38(70.4)$ & \\
\hline Female & $14(22.6)$ & $16(29.6)$ & 0.376 \\
\hline \multicolumn{4}{|l|}{ Smoking status } \\
\hline Smokers (current and past) & $19(30.6)$ & $8(14.8)$ & \\
\hline Non-smokers & $43(69.4)$ & $46(85.2)$ & $0.028 *$ \\
\hline \multicolumn{4}{|l|}{ Pneumothorax size $(\mathrm{cm})$} \\
\hline Initial size at presentation (mean) & 13.4 & 13.9 & 0.778 \\
\hline Collins $<4 \mathrm{~cm}(\mathrm{n})$ & $13(21.0)$ & $9(16.7)$ & \\
\hline Collins $\geq 4$ cm (n) & $47(75.8)$ & $42(77.8)$ & \\
\hline Missing (n) & $2(3.2)$ & $3(5.5)$ & 0.527 \\
\hline \multicolumn{4}{|l|}{ Age $(y)$} \\
\hline Median (Mean) & $23(24.6)$ & 24.(25.3) & 0.537 \\
\hline \multicolumn{4}{|l|}{ Height (m) } \\
\hline Median (Mean) & $1.8(1.8)$ & $1.8(1.8)$ & 1.000 \\
\hline \multicolumn{4}{|l|}{ Weight (kg) } \\
\hline Median (Mean) & $64.5(67.1)$ & $70(68.6)$ & 0.332 \\
\hline \multicolumn{4}{|l|}{ BMI $\left(\mathrm{kg} / \mathrm{m}^{2}\right)$} \\
\hline Median (Mean) & $20.5(20.7)$ & $21.4(21.0)$ & 0.348 \\
\hline \multicolumn{4}{|l|}{ Length of hospital stay (LOS) (days) } \\
\hline Mean (range) & $6.1(3-13)$ & $4.8(2-7)$ & $<0.001 *$ \\
\hline \multicolumn{4}{|l|}{ Days until operation } \\
\hline Mean (range) & $4.1(0-11)$ & / & / \\
\hline \multicolumn{4}{|l|}{ Operation time (min) } \\
\hline Mean (range) & $79.4(45-130)$ & / & / \\
\hline \multicolumn{4}{|l|}{ Chest tube duration (days) } \\
\hline Mean (range) & $5.5(3-8)$ & $5.2(2-6)$ & 0.836 \\
\hline \multicolumn{4}{|l|}{ Time until recurrence (months) } \\
\hline Mean (range) & $59(6-110)$ & $9.6(2-26)$ & $<0.001 *$ \\
\hline \multicolumn{4}{|l|}{ Complications (n) } \\
\hline Hemothorax & $3(4.8)$ & $0(0.0 \%)$ & 0.103 \\
\hline Prolonged air leak after surgery & $24(38.7)$ & / & / \\
\hline
\end{tabular}

During a median follow-up period of 76.5 months (range 1-155 months), patients who underwent $\mathrm{CT}$ treatment experienced a significantly higher recurrence rate compared with patients following VBPP (VBPP vs. CT: $9.7 \%$ vs. $64.8 \% ; p<0.0001$ ). This high rate of recurrence in the CT group occurred mainly within the first year after treatment (CT vs. VBPP: 9.6 months (range 2-26) vs. 59 months (range 6-110); $p<0.001$ ). Interestingly, patients with a large pneumothorax size (Collins $\geq 4 \mathrm{~cm}$ ) suffered a significantly higher rate of recurrence compared with patients with a small pneumothorax size (Collins $<4 \mathrm{~cm}$ vs. Collins $\geq 4 \mathrm{~cm}$ : $9.1 \%$ vs. $37 \%$; $p=0.010$ ) (Table 2 ). 
Table 2. Patient clinical characteristics and recurrence rates of PSP.

\begin{tabular}{|c|c|c|}
\hline Variable & $\begin{array}{c}\text { Recurrence } \\
n(\%)\end{array}$ & $p$-Value \\
\hline \multicolumn{3}{|l|}{ Gender } \\
\hline Male & $29(33.7)$ & 0.323 \\
\hline Female & $12(40.0)$ & \\
\hline \multicolumn{3}{|l|}{ Age } \\
\hline$\leq 24$ years & $22(33.4)$ & 0.756 \\
\hline >24 years & 19 (36.5) & \\
\hline \multicolumn{3}{|l|}{ BMI } \\
\hline$\leq 20.85 \mathrm{~kg} / \mathrm{m}^{2}$ & $20(35.5)$ & 0.843 \\
\hline$>20.85 \mathrm{~kg} / \mathrm{m}^{2}$ & $21(36.2)$ & \\
\hline \multicolumn{3}{|l|}{ Smoking status } \\
\hline Smokers (current and past) & $6(22.2)$ & 0.186 \\
\hline Non-smokers & $35(39.3)$ & \\
\hline \multicolumn{3}{|l|}{ Treatment } \\
\hline VBPP & $6(9.7)$ & $<0.0001$ * \\
\hline $\mathrm{CT}$ & $35(64.8)$ & \\
\hline \multicolumn{3}{|l|}{ Pneumothorax size $(\mathrm{cm})$} \\
\hline Collins $<4 \mathrm{~cm}$ & $2(9.1)$ & $0.010 *$ \\
\hline Collins $\geq 4 \mathrm{~cm}$ & 37 (41.6) & \\
\hline
\end{tabular}

Kg, kilogram; m, meter, VBPP, VATS bullectomy with partial pleurectomy; CT, chest tube; BMI, body mass index ${ }^{*} p$-value $<0.05$ indicates statistical significance.

Next, we investigated potential risk factors for recurrent pneumothorax in our patient cohort. Univariate analysis revealed that treatment of PSP patients with VBPP (VBPP vs. CT: HR 0.056; CI: $0.023-0.14 ; p<0.001$ ) and a small size of the initial pneumothorax (Collins $\geq 4$ cm vs. Collins $<4$ cm: HR 4.602; CI: 1.106-19.151; $p=0.020$ ) were significantly associated with a lower risk of pneumothorax recurrence (Table 3; Figure 1A,B). Both factors, namely the therapeutic procedure chosen (VBPP vs. CT: HR 0.047; CI: 0.017-0.132; $p<0.001$ ) and the baseline pneumothorax size (Collins $\geq 4 \mathrm{~cm}$ vs. Collins $<4 \mathrm{~cm}$ : HR 6.325; CI: 1.372-29.162; $p=0.018$ ), were confirmed as independent predictive markers of pneumothorax recurrence in multivariate regression analysis (Table 4).

Table 3. Univariate analysis of potential risk factors for recurrence of PSP.

\begin{tabular}{|c|c|c|c|}
\hline Risk Factor & Hazard Ratio & $95 \%$ CI & $p$-Value \\
\hline \multicolumn{4}{|l|}{ Gender } \\
\hline Male vs. female & 0.864 & $0.44-1.695$ & 0.668 \\
\hline \multicolumn{4}{|l|}{ Age } \\
\hline$>$ median vs. $\leq$ median & 1.088 & $0.588-2.011$ & 0.787 \\
\hline \multicolumn{4}{|l|}{ BMI } \\
\hline$>$ median vs. $\leq$ median & 1.155 & $0.623-2.139$ & 0.644 \\
\hline \multicolumn{4}{|l|}{ Smoking status } \\
\hline Smoker vs. non-smokers & 0.536 & $0.220-1.246$ & 0.133 \\
\hline \multicolumn{4}{|l|}{ Treatment } \\
\hline VBPP vs. CT & 0.056 & $0.023-0.14$ & $<0.001 *$ \\
\hline \multicolumn{4}{|l|}{ Pneumothorax size } \\
\hline Collins $\geq 4$ vs. Collins $<4$ & 4.602 & $1.106-19.151$ & $0.020 *$ \\
\hline
\end{tabular}

Univariate analysis displays potential risk factors that might influence recurrence-free survival (RFS). Patients with a large pneumothorax size (Collin $\geq 4$ ) and those treated by chest tube (CT) have a significantly low RFS * $p$-value $<0.05$ indicates statistical significance. VBPP, VATS bullectomy with partial pleurectomy; BMI, body mass index; $\mathrm{CI}$, confidential interval. 


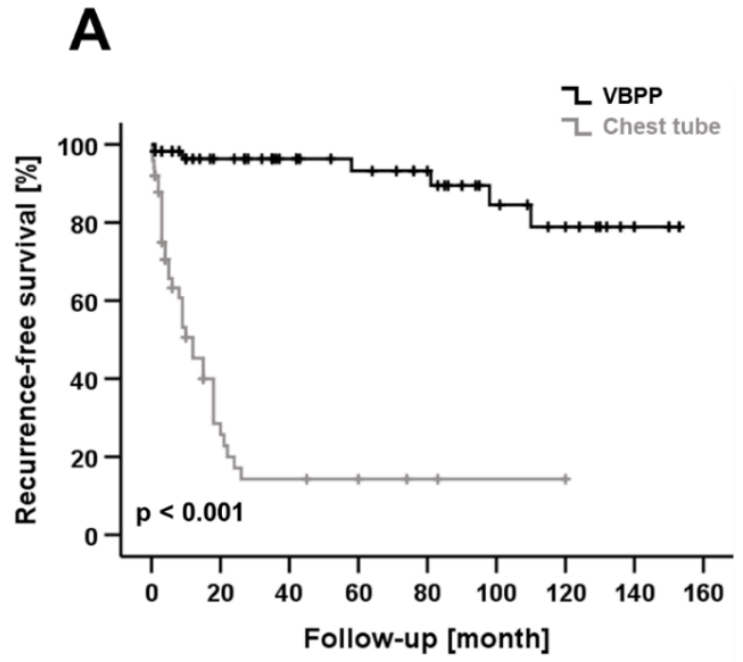

B

Figure 1. (A) Kaplan-Meier curve shows recurrence-free survival (RFS) after treatment by surgery (VBPP) or chest tube (CT). VBPP was associated with significantly better RFS compared to CT treatment. (B) Patients with a large pneumothorax size (Collins $\geq 4 \mathrm{~cm}$ ) had a significantly reduced RFS compared to patients with a small pneumothorax size (Collins $<4 \mathrm{~cm}$ ).

Table 4. Multivariate analysis of potential risk factors for recurrence of PSP.

\begin{tabular}{cccc}
\hline \multicolumn{1}{c}{ Risk Factor } & Hazard Ratio & $\mathbf{9 5 \%}$ CI & $p$-Value \\
\hline $\begin{array}{l}\text { Treatment } \\
\text { VBPP vs. CT }\end{array}$ & 0.047 & $0.017-0.132$ & $<0.001$ * \\
$\begin{array}{l}\text { Pneumothorax size } \\
\text { Collins } \geq 4 \text { vs. Collins }<4\end{array}$ & 6.325 & $1.372-29.162$ & $0.018^{*}$ \\
\hline
\end{tabular}

Multivariate analysis displayed initial pneumothorax size and administered treatment as independent risk factors for PSP recurrence. Chest tube (CT) treatment and a large pneumothorax size (Collins $\geq 4)$ were associated with a significantly reduced recurrence-free survival. ${ }^{*} p$-value $<0.05$ indicates statistical significance. VBPP, VATS bullectomy with partial pleurectomy; CI, confidential interval.

\section{Discussion}

Nowadays, VATS-bullectomy with partial pleurectomy (VBPP) is a well-established and increasingly used surgical treatment for primary spontaneous pneumothorax (PSP). Recently, VBPP has been reported with superior performance and low rates of recurrence compared to VATS bullectomy alone $[20,21,24,25]$. However, only a few studies elucidate its effectiveness in terms of long-term outcomes. Additionally, studies that examine potential risk factors for PSP recurrence following surgical treatment are rare. In this study, we evaluated the long-term recurrence rates of patients treated with VBPP or CT in our institution. Moreover, we examined underlying clinical factors that might influence disease recurrence in our patient cohort.

In our study, 62 patients with a mean age of 24.6 years underwent VBPP, while 54 patients (mean age 25.3 years) (Table 1) were successfully treated by chest tube. Compared to some previous studies in the literature with a high number of smokers in their PSP collective $[12,16,17]$, we had a significantly low number of smokers in both groups of our patient cohort (Table 1). This might be due to the increased awareness campaigns and information about the negative effects of smoking on human health over the last years. Interestingly, the length of hospital stay (LOS) of patients in the VBPP group was significantly longer than in the CT group. Although the LOS in the VBPP group was consistent with previous studies reporting the hospitalization time after VATS in the literature $[27,28]$, this result was significantly prolonged by the number of hospital days until surgery was performed (Table 1).

During a follow-up period of 76.5 months (range 1-155 months), six (9.7\%) patients suffered a recurrence following VBPP. Compared to the VBPP group, the CT group experienced 
a higher recurrence rate of $64.8 \%$ (35 patients recurred, Table 2). This difference was statistically significant in the univariate (VBPP vs. CT: HR 0.056; CI: $0.023-0.14 ; p<0.001$ ) and multivariate analyses (VBPP vs. CT: HR 0.047; CI: 0.017-0.132; $p<0.001$ ) (Tables 3 and 4). These results demonstrate that VBPP is associated with an increased recurrence-free survival compared to CT treatment. The recurrence rate of $9.7 \%$ of our VBPP cohort is slightly higher than some studies described in the literature. In the study of Imperatori, A. et al. [23] with 134 patients, VATS blebectomy and parietal pleurectomy had a recurrence rate of $6 \%$. The median follow-up was 79 months (range: 36-187 months). Shaikhrezai, K et al. [24] observed no recurrent pneumothorax of 44 PSP patients operated by VATS bullectomy and partial pleurectomy during a median follow-up of 73 months. In a recent study of Caecilia $\mathrm{Ng}$ et al. [25], in which 73 PSP patients underwent VATS partial pleurectomy, a recurrence rate of $1.4 \%$ at a median follow-up period of 58.5 months was reported. Although the above recurrence rates are lower than the recurrence rate of our VBPP cohort, none of them evaluated the pneumothorax size as a potential risk factor for PSP recurrence. In our study, the patients presented with a large pneumothorax size estimated according to the method of Collins. The mean pneumothorax size of the VBPP group was $13.4 \mathrm{~cm}$ and $13.9 \mathrm{~cm}$ for the $\mathrm{CT}$ group. As reported in earlier studies, a large pneumothorax size is a risk factor for PSP recurrence $[9,29,30]$. Therefore, we assumed that the large pneumothorax size of our PSP cohort influenced our comparatively high rate of recurrence.

To determine potential risk factors for recurrence, we analyzed the impact of clinical factors, such as gender, age, BMI, smoking status, pneumothorax size, and the treatment modality on PSP recurrence. Treatment by CT placement (VBPP vs. CT: HR 0.047; CI: $0.017-0.132 ; p<0.001$ ) and a large pneumothorax size (Collins $\geq 4 \mathrm{~cm}$ vs. Collins $<4 \mathrm{~cm}$ : HR 6.325; CI: 1.372-29.162; $p=0.018$ ) proved to be independent risk factors for disease recurrence in our PSP patient cohort. These results highlight the high efficacy of VBPP in the treatment of patients with PSP and the clinical importance of the initial pneumothorax size as a significant risk factor for disease recurrence. Noteworthy, other potential risk factors for disease recurrence, such as family history of pneumothorax, presence of connective tissue disorders, cannabis consumption, and scoliosis, were also investigated. However, the small number of patients with these risk factors limited the statistical analysis, and thus, they were not included as risk factors in this study.

The indication to perform surgery (VBPP) might differ internationally. However, our data demonstrate that the risk of recurrent pneumothorax can be significantly reduced by VBPP. In addition, our data highlight the fact that a large initial pneumothorax size is associated with a markedly increased risk of pneumothorax recurrence. Obviously, the power of our study is limited due to its retrospective design and the small number of patients included, but the logical consequence based on our observations would be to prefer VBPP to simple chest drainage in patients with a large pneumothorax size. However, this observation should be verified in large prospective randomized trials.

\section{Conclusions}

Our data confirm that VBPP is an effective and safe surgical treatment for PSP. It is associated with a very low risk of disease recurrence. A large pneumothorax size is an independent risk factor for recurrence of PSP. Therefore, the option of VBPP should be discussed early for PSP patients treated with a large pneumothorax size, and close follow-ups should be performed due to the high risk of recurrence.

Author Contributions: Conceptualization, S.F., H.A., M.K., S.-A.S., A.S., A.K., M.S., F.Z., A.R., L.D. and W.-T.K.; methodology, S.F., H.A., M.K., A.R., F.Z., L.D. and W.-T.K.; software, S.F., L.D. and F.Z.; validation, S.F., H.A., M.K., S.-A.S., A.S., A.K., M.S., F.Z., A.R., L.D. and W.-T.K.; formal analysis, S.F., H.A., M.S., L.D. and W.-T.K.; investigation, S.F., H.A., M.S., L.D. and W.-T.K.; data curation, S.F., H.A., M.K. and L.D.; writing-original draft preparation, S.F., M.K. and L.D.; writing—review and editing, S.F., M.S., A.S., A.K., S.-A.S., L.D. and W.-T.K.; visualization, S.F. and L.D.; supervision, S.F., M.K., A.R., L.D. and W.-T.K.; project administration, S.F., M.K. and L.D. All authors have read and agreed to the published version of the manuscript. 
Funding: This research received no external funding.

Institutional Review Board Statement: The study was conducted according to the guidelines of the Declaration of Helsinki and approved by the local institutional review board of the Heinrich Heine University Clinic, Duesseldorf, Germany; Study Nr: 2020-1271.

Informed Consent Statement: Informed consent to participate was waived because no data regarding the cases were disclosed.

Data Availability Statement: The data presented are included in this study; the corresponding author on request may provide additional data.

Conflicts of Interest: The authors declare no conflict of interest.

\section{References}

1. Schnell, J.; Beer, M.; Eggeling, S.; Gesierich, W.; Gottlieb, J.; Herth, F.J.F.; Hofmann, H.S.; Jany, B.; Kreuter, M.; Ley-Zaporozhan, J.; et al. Management of Spontaneous Pneumothorax and Post-Interventional Pneumothorax: German S3 Guideline. Respiration 2019, 97, 370-402. [CrossRef] [PubMed]

2. Sahn, S.A.; Heffner, J.E. Spontaneous pneumothorax. N. Engl. J. Med. 2000, 342, 868-874. [CrossRef] [PubMed]

3. Noppen, M. Management of primary spontaneous pneumothorax. Curr. Opin. Pulm. Med. 2003, 9, 272-275. [CrossRef] [PubMed]

4. Lyra Rde, M. Etiology of primary spontaneous pneumothorax. J. Bras. Pneumol. 2016, 42, 222-226. [CrossRef] [PubMed]

5. Primrose, W.R. Spontaneous pneumothorax: A retrospective review of aetiology, pathogenesis and management. Scott. Med. J. 1984, 29, 15-20. [CrossRef] [PubMed]

6. Bense, L.; Eklund, G.; Wiman, L.G. Smoking and the increased risk of contracting spontaneous pneumothorax. Chest 1987, 92, 1009-1012. [CrossRef] [PubMed]

7. Gupta, D.; Hansell, A.; Nichols, T.; Duong, T.; Ayres, J.G.; Strachan, D. Epidemiology of pneumothorax in England. Thorax 2000, 55, 666-671. [CrossRef]

8. Sadikot, R.T.; Greene, T.; Meadows, K.; Arnold, A.G. Recurrence of primary spontaneous pneumothorax. Thorax 1997, 52, 805-809. [CrossRef]

9. Sayar, A.; Kök, A.; Citak, N.; Metin, M.; Büyükkale, S.; Gürses, A. Size of pneumothorax can be a new indication for surgical treatment in primary spontaneous pneumothorax: A prospective study. Ann. Thorac. Cardiovasc. Surg. 2014, 20, 192-197. [CrossRef]

10. MacDuff, A.; Arnold, A.; Harvey, J. Management of spontaneous pneumothorax: British Thoracic Society Pleural Disease Guideline 2010. Thorax 2010, 65 (Suppl. S2), ii18-ii31. [CrossRef]

11. Tschopp, J.M.; Bintcliffe, O.; Astoul, P.; Canalis, E.; Driesen, P.; Janssen, J.; Krasnik, M.; Maskell, N.; Van Schil, P.; Tonia, T.; et al. ERS task force statement: Diagnosis and treatment of primary spontaneous pneumothorax. Eur. Respir. J. 2015, 46, 321-335. [CrossRef]

12. Brown, S.G.A.; Ball, E.L.; Perrin, K.; Asha, S.E.; Braithwaite, I.; Egerton-Warburton, D.; Jones, P.G.; Keijzers, G.; Kinnear, F.B.; Kwan, B.C.H.; et al. Conservative versus Interventional Treatment for Spontaneous Pneumothorax. N. Engl. J. Med. 2020, 382, 405-415. [CrossRef]

13. Ennis, S.L.; Dobler, C.C. Conservative versus interventional treatment for spontaneous pneumothorax. Breathe 2020, 16, 200171. [CrossRef]

14. Noppen, M.; Alexander, P.; Driesen, P.; Slabbynck, H.; Verstraeten, A. Manual aspiration versus chest tube drainage in first episodes of primary spontaneous pneumothorax: A multicenter, prospective, randomized pilot study. Am. J. Respir. Crit. Care Med. 2002, 165, 1240-1244. [CrossRef]

15. Ayed, A.K.; Chandrasekaran, C.; Sukumar, M. Aspiration versus tube drainage in primary spontaneous pneumothorax: A randomised study. Eur. Respir. J. 2006, 27, 477-482. [CrossRef]

16. Olesen, W.H.; Lindahl-Jacobsen, R.; Katballe, N.; Sindby, J.E.; Titlestad, I.L.; Andersen, P.E.; Licht, P.B. Recurrent Primary Spontaneous Pneumothorax is Common Following Chest Tube and Conservative Treatment. World J. Surg. 2016, 40, 2163-2170. [CrossRef]

17. Olesen, W.H.; Katballe, N.; Sindby, J.E.; Titlestad, I.L.; Andersen, P.E.; Lindahl-Jacobsen, R.; Licht, P.B. Surgical treatment versus conventional chest tube drainage in primary spontaneous pneumothorax: A randomized controlled trial. Eur. J. Cardio-Thorac. Surg. Off. J. Eur. Assoc. Cardio-Thorac. Surg. 2018, 54, 113-121. [CrossRef]

18. Muramatsu, T.; Ohmori, K.; Shimamura, M.; Furuichi, M.; Takeshita, S.; Negishi, N. Staple line reinforcement with fleece-coated fibrin glue (TachoComb) after thoracoscopic bullectomy for the treatment of spontaneous pneumothorax. Surg. Today 2007, 37, 745-749. [CrossRef]

19. Nakanishi, K. Long-term effect of a thoracoscopic stapled bullectomy alone for preventing the recurrence of primary spontaneous pneumothorax. Surg. Today 2009, 39, 553-557. [CrossRef]

20. Mithiran, H.; Leow, L.; Ong, K.; Liew, T.; Siva, D.; Liang, S.; Tam, J.K.C. Video-Assisted Thoracic Surgery (VATS) Talc Pleurodesis Versus Pleurectomy for Primary Spontaneous Pneumothorax: A Large Single-Centre Study with No Conversion. World J. Surg. 2019, 43, 2099-2105. [CrossRef] 
21. Neudecker, J.; Malzahn, U.; Heuschmann, P.; Behrens, U.; Walles, T. Pulmonary wedge resection plus parietal pleurectomy (WRPP) versus parietal pleurectomy (PP) for the treatment of recurrent primary pneumothorax (WOPP trial): Study protocol for a randomized controlled trial. Trials 2015, 16, 540. [CrossRef] [PubMed]

22. Czerny, M.; Salat, A.; Fleck, T.; Hofmann, W.; Zimpfer, D.; Eckersberger, F.; Klepetko, W.; Wolner, E.; Mueller, M.R. Lung wedge resection improves outcome in stage I primary spontaneous pneumothorax. Ann. Thorac. Surg. 2004, 77, 1802-1805. [CrossRef] [PubMed]

23. Imperatori, A.; Rotolo, N.; Spagnoletti, M.; Festi, L.; Berizzi, F.; Di Natale, D.; Nardecchia, E.; Dominioni, L. Risk factors for postoperative recurrence of spontaneous pneumothorax treated by video-assisted thoracoscopic surgery + . Interact. Cardiovasc. Thorac. Surg. 2015, 20, 647-651. [CrossRef] [PubMed]

24. Shaikhrezai, K.; Thompson, A.I.; Parkin, C.; Stamenkovic, S.; Walker, W.S. Video-assisted thoracoscopic surgery management of spontaneous pneumothorax-long-term results. Eur. J. Cardio-Thorac. Surg. Off. J. Eur. Assoc. Cardio-Thorac. Surg. 2011, 40, 120-123. [CrossRef]

25. Ng, C.; Maier, H.T.; Kocher, F.; Jud, S.; Lucciarini, P.; Öfner, D.; Schmid, T.; Augustin, F. VATS Partial Pleurectomy Versus VATS Pleural Abrasion: Significant Reduction in Pneumothorax Recurrence Rates After Pleurectomy. World J. Surg. 2018, 42, 3256-3262. [CrossRef]

26. Collins, C.D.; Lopez, A.; Mathie, A.; Wood, V.; Jackson, J.E.; Roddie, M.E. Quantification of pneumothorax size on chest radiographs using interpleural distances: Regression analysis based on volume measurements from helical CT. AJR Am. J. Roentgenol. 1995, 165, 1127-1130. [CrossRef]

27. Herrmann, D.; Klapdor, B.; Ewig, S.; Hecker, E. Initial management of primary spontaneous pneumothorax with video-assisted thoracoscopic surgery: A 10-year experience. Eur. J. Cardio-Thorac. Surg. Off. J. Eur. Assoc. Cardio-Thorac. Surg. 2016, 49, 854-859. [CrossRef]

28. Delpy, J.P.; Pagès, P.B.; Mordant, P.; Falcoz, P.E.; Thomas, P.; Le Pimpec-Barthes, F.; Dahan, M.; Bernard, A. Surgical management of spontaneous pneumothorax: Are there any prognostic factors influencing postoperative complications? Eur. J. Cardio-Thorac. Surg. Off. J. Eur. Assoc. Cardio-Thorac. Surg. 2016, 49, 862-867. [CrossRef]

29. Tan, J.; Yang, Y.; Zhong, J.; Zuo, C.; Tang, H.; Zhao, H.; Zeng, G.; Zhang, J.; Guo, J.; Yang, N. Association Between BMI and Recurrence of Primary Spontaneous Pneumothorax. World J. Surg. 2017, 41, 1274-1280. [CrossRef]

30. Tsai, T.M.; Lin, M.W.; Li, Y.J.; Chang, C.H.; Liao, H.C.; Liu, C.Y.; Hsu, H.H.; Chen, J.S. The Size of Spontaneous Pneumothorax is a Predictor of Unsuccessful Catheter Drainage. Sci. Rep. 2017, 7, 181. [CrossRef] 